\title{
Reshaping the eyebrow by follicular unit transplantation from excised eyebrow in extended infrabrow excision blepharoplasty
}

This article was published in the following Dove Press journal:

Clinical Ophthalmology

13 February20I2

Number of times this article has been viewed

\author{
Kazuhito Konishi' \\ Isao Sugimoto ${ }^{2}$ \\ Hirohiko Kakizaki ${ }^{3}$ \\ Akihiro Ichinose ${ }^{4}$ \\ 'Kobe Academia Clinic, ${ }^{2}$ Division \\ of Aesthetic Medical Science, \\ Department of Plastic Surgery, \\ Kobe University Graduate School \\ of Medicine, ${ }^{3}$ Department of \\ Ophthalmology, Aichi Medical \\ University, Nagoya, Aichi, Japan; \\ ${ }^{4}$ Department of Plastic Surgery, \\ Graduate School of Medicine, Kobe \\ University, Kobe, Hyogo, Japan
}

Purpose: We report the reshaping of the eyebrow by follicular unit transplantation from excised eyebrow skin in extended infrabrow excision blepharoplasty.

Methods: The method was carried out in two patients with moderate or significant dermatochalasis. The areas to be excised from the infrabrow and intrabrow skin were decided upon and the area of hair transplantation was planned in the suprabrow area and the tail of the eyebrow. The skin was removed and the excised intrabrow skin was dissected into single follicular units. Tiny incisions were made with a scalpel, and grafts were inserted using fine forceps.

Results: Almost all transplanted eyebrow follicles took successfully, resulting in eyebrows of a desirable shape as planned preoperatively. The reshaped eyebrows had a natural appearance since the transplanted hairs were similar to those of the original eyebrow. The eyelids still looked youthful rather than operated-on. Finally, the evidence of blepharoplasty was hardly visible and patient satisfaction was quite high. The patients felt more ease in opening their eyes and obtained a wider visual field.

Conclusion: Our study demonstrated excellent results with hair transplantation from eyebrow to eyebrow, a finding which, to our knowledge, has not been previously reported. Our method can be used for selected patients, especially men, with moderate to severe dermatochalasis, who find a reduction or change in the shape of the eyebrow unacceptable.

Keywords: blepharoplasty, dermatochalasis, follicular unit transplantation, eyebrow reconstruction

\section{Introduction}

Infrabrow excision blepharoplasty (IBEB) was originally reported as an option for mild dermatochalasis in Caucasians. ${ }^{1-3,5}$ Since the procedure is very simple and fairly natural rejuvenation of the eyelid is possible, from the 1990s its usage has expanded in Asians, ${ }^{4,6,7}$ replacing conventional blepharoplasty. ${ }^{8}$ Today, IBEB has become a prevalent option for mild dermatochalasis in Asians. ${ }^{9}$ However, this method is not a good option for patients with significant dermatochalasis or dermatochalasis with eyebrow ptosis. Therefore, we have reported extended infrabrow excision blepharoplasty (extended IBEB), ${ }^{10}$ where the area of skin excision is extended to the intrabrow portion. The incision of intrabrow skin perpendicular to the hair shafts allows hair regrowth through the scar, thus contributing to making the scar less conspicuous and alleviating the reduction of the eyebrow. This method is indicated for middle-aged or elderly patients with significant dermatochalasis, but it has not been indicated for patients, especially men, who find any postoperative reduction or change in the shape of the eyebrow unacceptable.
Correspondence: Kazuhito Konishi

3 chome I-13, Isogami-dori,

Chuo-ku, Kobe, Hyogo, Japan

$\mathrm{Tel}+8 \mathrm{I} 782656524$

Fax +8I 782656724

Email kkkk1977@me.com 
In this paper, we present extended IBEB, reshaping the eyebrow with follicular unit transplantation (FUT) from the excised eyebrow (extended IBEB with FUT). This can give the eyebrows a cosmetically desirable shape after extended IBEB.

\section{Methods}

\section{Preoperative planning and marking}

For this method, we considered patients in whom no change of the shape of the eyebrow was desirable - particularly men who have significant dermatochalasis.

The tear-shaped skin pad to be excised in the infrabrow and intrabrow areas is marked with the patient in the supine position (Figure 1). The lateral point ends $10 \mathrm{~mm}$ laterally out from the tail of the eyebrow. The skin pad includes a substantial amount of the lateral portion and some of the medial portion of the brow. The skin pad is $10-20 \mathrm{~mm}$ at its greatest vertical width.

The outline of the area of hair transplantation is carefully marked according to the design agreed upon during consultation with the patient.

Many patients with dermatochalasis raise the eyebrows excessively, thus it is often found that the eyebrows decline postoperatively due to relaxation of the frontal muscle (Figure 2). Consequently, in many cases the transplantation area is designed in the suprabrow region to match the shape of the original eyebrow, with good results (Figure 3 ).

\section{Operative technique}

Surgery is carried out under local anesthesia with a solution of 2\% lidocaine with 1:100000 epinephrine. In addition, a small amount of anesthetic is injected into the supratrochlear and supraorbital notches to create a nerve block.
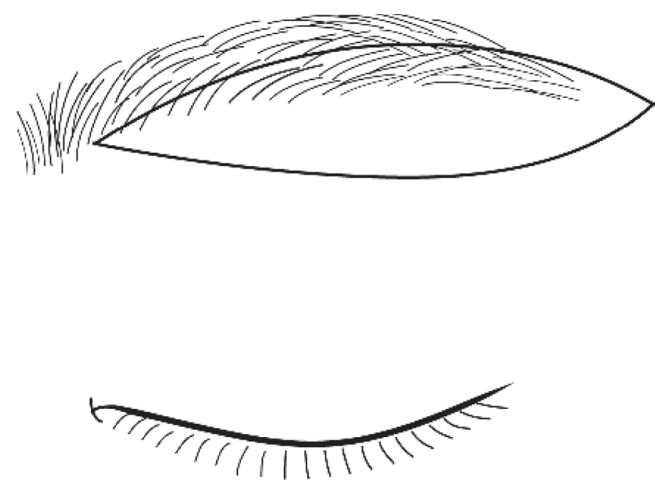

Figure I Design of extended IBEB. The medial end of the excised skin pad is $5 \mathrm{~mm}$ laterally in from the start of the eyebrow, and the lateral end of the skin pad is $5-10 \mathrm{~mm}$ laterally out from the tail of the eyebrow. The incision is made perpendicularly to the hair shafts. The vertical width of the skin pad is $10-20 \mathrm{~mm}$ at its widest point in most patients.
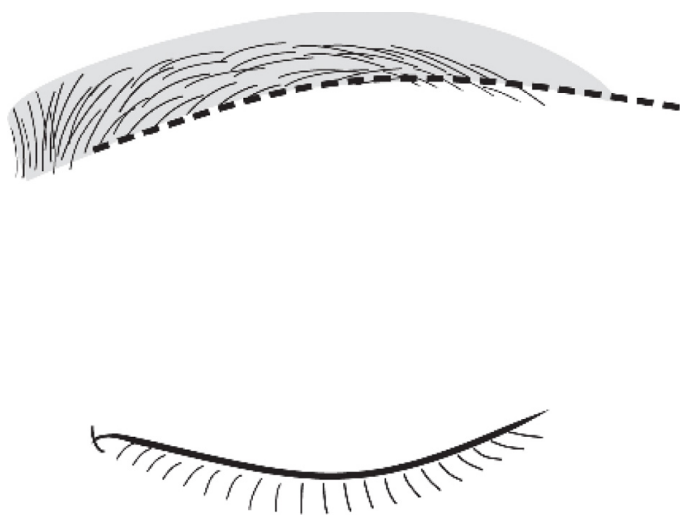

Figure 2 Planning the desirable postoperative eyebrow shape. The desirable eyebrow shape after surgery is shown (gray area). Postoperative descent of the eyebrow, which is generally observed after extended IBEB, has to be considered. It is unnecessary to transplant hair into the infrabrow operative scar (dotted line), as it would be covered by the regrowth of hair, made possible by incision perpendicular to the hair shafts.

The skin excision is performed as reported by Ichinose et al. ${ }^{10}$ The skin pad is excised from the layer above the orbicularis oculi. Several dermal stitches are made with 5-0 polydioxanone sutures. The skin is closed with 6-0 black nylon so that the edges of both wound margins match precisely.

Excised skin is placed under a series of dissecting microscopes, where the individual follicular units are dissected into grafts. Each graft consists of one follicular unit. Hairless skin and fat between follicular units is removed carefully. Tiny incisions are made with a scalpel, and the grafts inserted using fine forceps.

The hair in the tail of the eyebrow points horizontally and in the upper part of the eyebrow points slightly downward. Therefore, proper angulation of the grafted follicles is most important.

No dressing is required after the operation. Patients are instructed to gently irrigate the transplanted area to remove
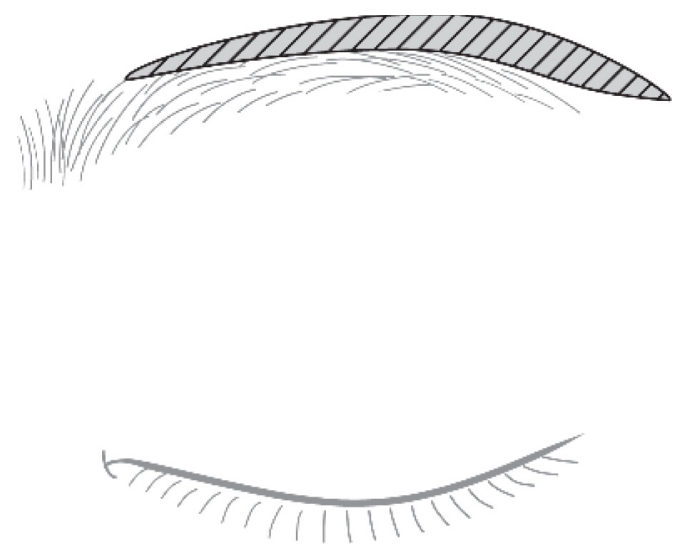

Figure 3 Marking of the area of hair transplantation. The area above the upper margin and the tail of the eyebrow (shadow area) is designed so that it will develop into the desired shape. 


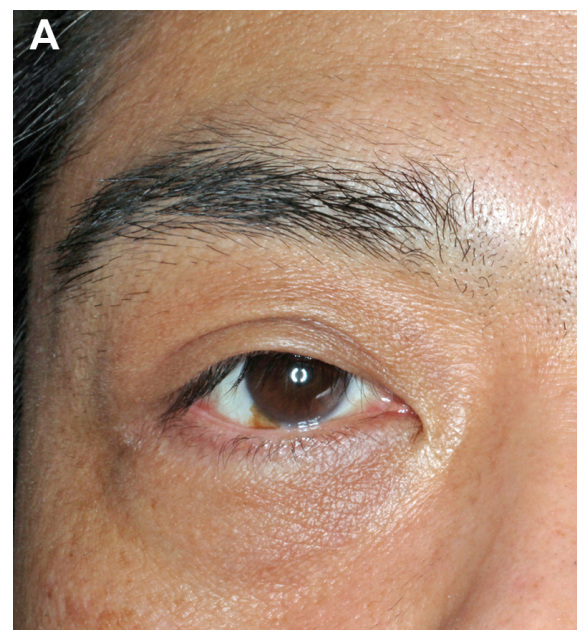

B

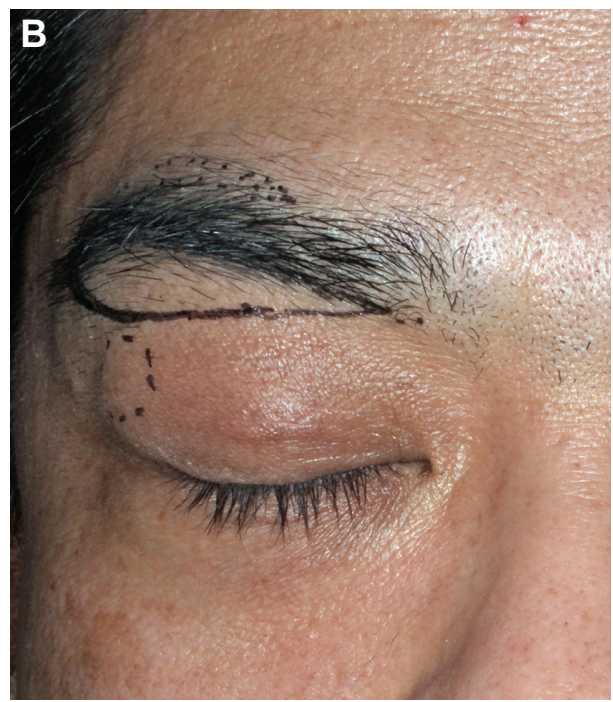

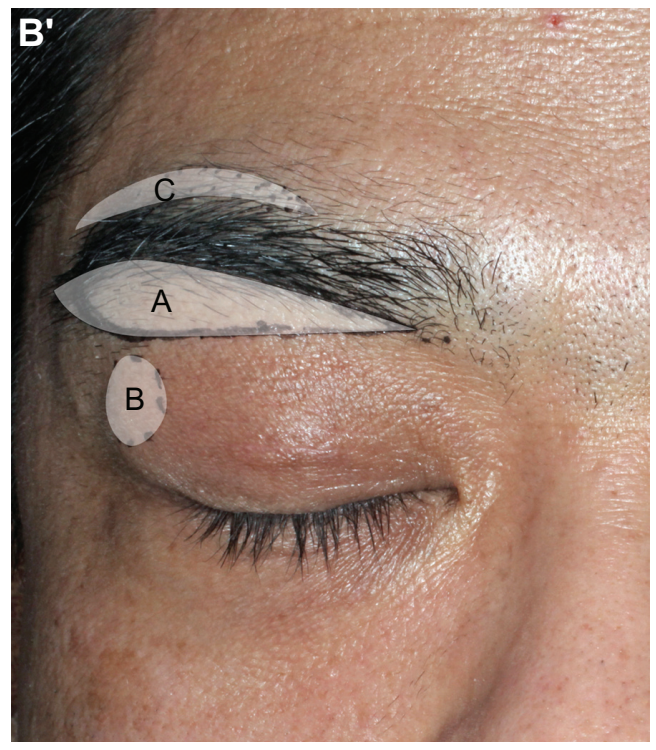

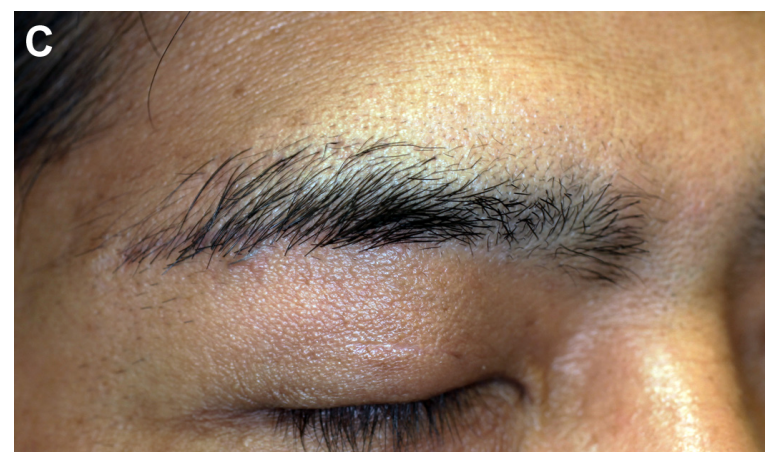

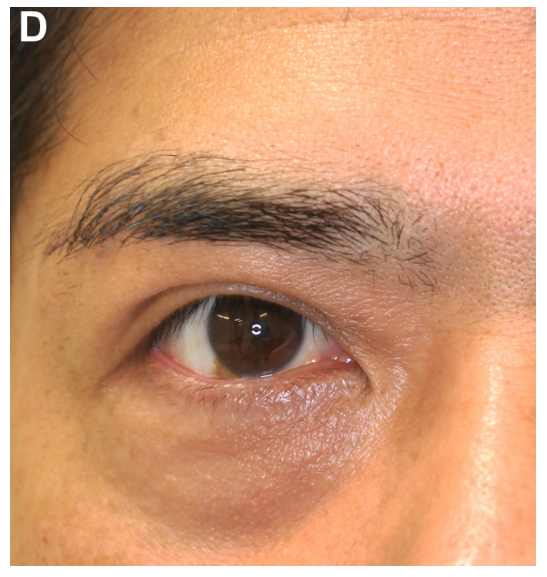

Figure 4 Case I. (A) A 50-year-old man with moderate bilateral dermatochalasis. (B) Extended IBEB with FUT was carried out. The maximum width of the skin pad was $17 \mathrm{~mm}$. The subseptal fat was dissected. The incision was made perpendicularly to the hair shafts. The numbers of follicular units available to transplant were 100 on each side, and these were transplanted in the upper part of the eyebrow from body to tail. (B') The portion of excised the eyebrow, is area A. Approximately half of the eyebrow was excised from the body to the tail of the eyebrow. The portion of removed orbital fat is area B. The portion of transplanted hair to the eyebrow is area C. It extended from the center of the eyebrow to the outside of the eyebrow's superior border with a maximum width of $7 \mathrm{~mm}$. (C) Twelve months after the operation, the scar had become less conspicuous. The scar just along the lower margin of the eyebrow was partially covered by regrown hair. The transplanted hair was growing. (D) Twenty-four months after the operation, the regrown eyebrow entirely covered the scar and made it inconspicuous. The transplanted hair was also fully grown. Any changes in, reduction, or distortion of the eyebrow were acceptable. The patient was satisfied with the result and no complications were observed. He opened his eyes more easily, his visual field had become larger, and his lid creases had a more youthful appearance. 

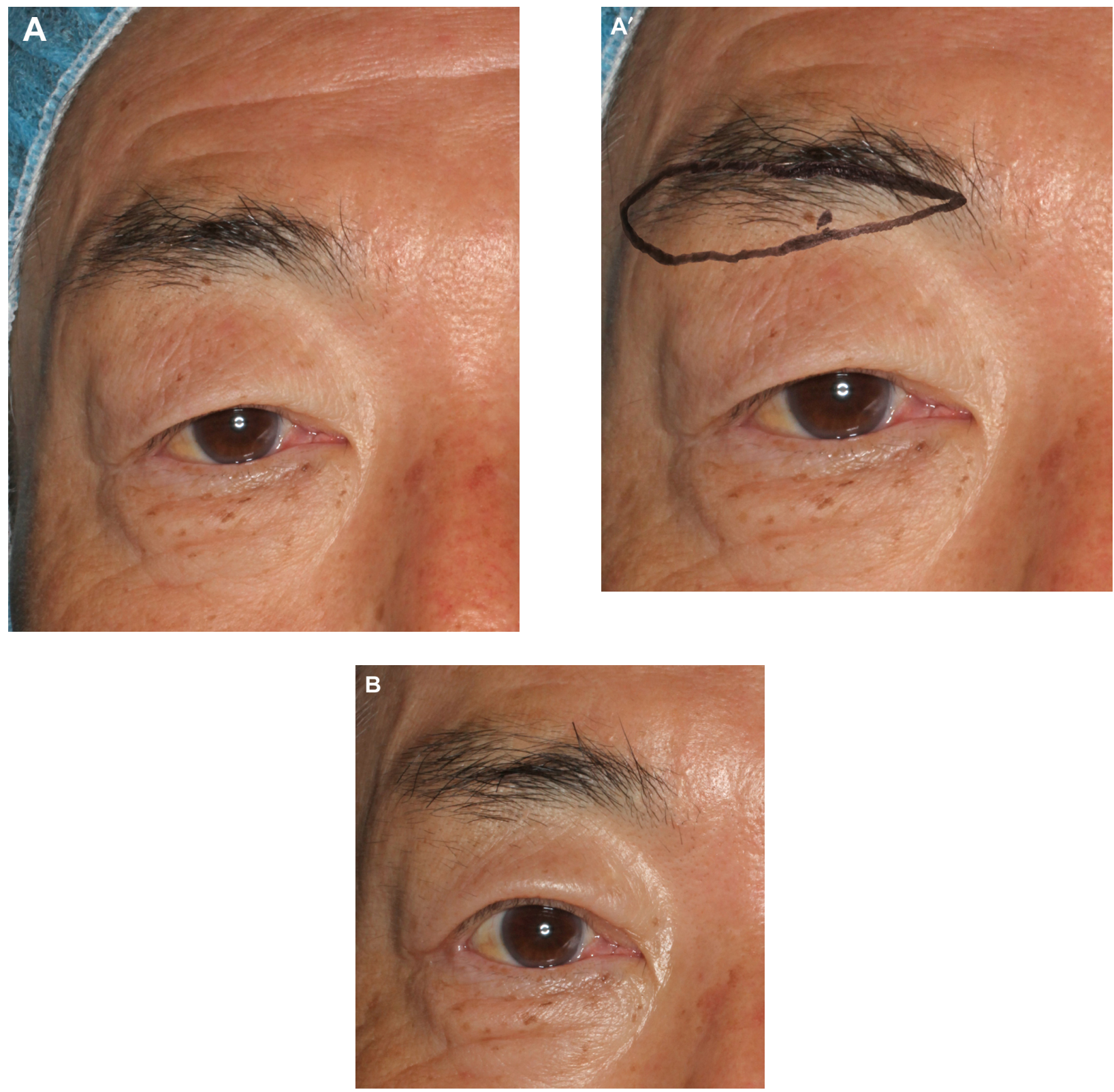

Figure 5 Case 2. (A) A 56-year-old man with severe bilateral dermatochalasis. He had a sleepy looking appearance due to droopy eyelids. Lateral hooding impaired the upper visual field. The maximum width of eyebrows was $1 \mathrm{~cm}$. (A') Extended IBEB with FUT was carried out, where the maximum width of the excised skin pad was 20 mm. The numbers of follicular units available to transplant were 150 on each side, and these were transplanted into the upper part of the eyebrow from body to tail. (B) Twelve months after the operation, the decline and reduction of the eyebrow was slight and the patient was able to open his eyes easily. The maximum width was not as variable as before the operation at $\mathrm{I} \mathrm{cm}$. He was satisfied with the result and no complications were observed.

any dried crusts in the shower twice daily for a week. After each shower, patients should apply an antibiotic ointment to the eyebrow.

\section{Patients}

The method was carried out in two patients with moderate and significant dermatochalasis. Both patients were men, aged 50 and 56 years. Their chief complaint was a tired feeling of the eyes caused by excess eyelid skin. Dermatochalasis resulted in a superior visual field defect.
The maximum width of the excised skin pads from the infrabrow and intrabrow areas was $17 \mathrm{~mm}$ and $20 \mathrm{~mm}$, respectively, and the numbers of follicular units transplanted were 100 and 150 on each side, respectively. They were transplanted into the skin above the eyebrow, from the body to the tail.

\section{Results}

By the end of the first month, most of the transplanted eyebrow hairs had fallen out. At around 3 months, the new 
eyebrow hair grafts had begun to grow. At 10 months postoperatively the final results of the eyebrow transplant procedure were apparent. Almost all transplanted hairs had taken. The transplanted hairs were very similar to the original hairs and the eyebrows were indistinguishable without comparing the pre- and postoperative photographs. The postoperative eyebrow shape that had been planned preoperatively was achieved. In addition, regrowth of the eyebrow, after incision perpendicular to the hair shafts, was observed through the infrabrow scar, covering the scar and making it more inconspicuous a few weeks postoperatively. This also alleviated the reduction of the eyebrow to some degree.

High patient satisfaction was achieved with respect to aesthetics. The patients' overall satisfaction levels on a fivestep evaluation scale were 4 and 5 , respectively. The operation resulted in natural eyebrows and eyelids with a youthful appearance and minimal signs of scarring or other adverse effects. Patient satisfaction with eyelid function was also high, since the patients experienced ease in opening their eyes and obtained a wider visual field. Photographs of the patients are shown in Figures 4 and 5.

\section{Discussion}

Our report has demonstrated that extended IBEB with FUT provides a significant reduction in the redundancy of the eyelid without undesirable deformation of the eyebrow. The eyebrow which has been reshaped by FUT using excised eyebrow is quite natural and signs that the patient has undergone blepharoplasty are hardly distinguishable. Furthermore, the incision of intrabrow skin perpendicular to the hair shafts allows hair regrowth through the scar. This covers the infrabrow scar, making it less conspicuous and alleviating the reduction of the eyebrow to some degree.

We have demonstrated excellent results with hair transplantation from eyebrow to eyebrow, a finding which has not been previously reported, to our knowledge. Eyebrow reconstruction is often carried out by scalp hair transplantation, ${ }^{11-19}$ but high patient satisfaction is not always achieved with this method. The implanted hair from the scalp is thicker than the hair of eyebrow. The patients also have to trim the eyebrows frequently since the hair cycle of the scalp is longer than that of the eyebrow.

The surgeon should treat the eyebrow follicles carefully as they are easily damaged. ${ }^{13}$ They should also give much consideration to the direction of the transplanted hair to make the eyebrow look natural. The direction of the hairs of an eyebrow does not follow a simple pattern but varies throughout the eyebrow. For example, the hair grows in a slightly downward direction in the upper part of the tail of the eyebrow while in a slightly upward direction in the lower part.

The drawback of using FUT is that it is a somewhat time-consuming procedure. Surgical time is extended by about 10 minutes when FUT is added to extended IBEB, to allow for dissection of follicular units into grafts by a skilled technician. This can limit the usage of the method.

The method can be used for some patients, especially men, for whom extended IBEB is indicated, but who will not accept any reduction or change in the shape of the eyebrow. It can also be used for patients with eyebrow ptosis, since the eyebrow can be reshaped in a higher position instead of being lifted. We hope this method becomes a useful option for the treatment of dermatochalasis in selected patients.

\section{Conclusion}

This report presents extended IBEB combined with reshaping of the eyebrow using FUT from the excised eyebrow. The method is effective in reducing eyelid redundancy especially in Asian male patients with severe dermatochalasis. The method is useful in selected patients, although in our experience FUT is not necessary in the majority of patients who undergo extended IBEB.

\section{Disclosure}

The authors have no proprietary interest in the materials presented herein.

\section{References}

1. Parkes ML, Kamer FM, Merrin M. Infrabrow lift. Laryngoscope. 1976;86(12):1869-1872.

2. Camirand A, Doucet J. Surgical advances: A comprehensive approach to surgical rejuvenation of the eyes. Aesthetic Plast Surg. 1996;20(1):15-22.

3. Widgerow AD. Upper blepharoplasty with lateral segmental orbicularis excision. Ann Plast Surg. 2003;50(5):471-474.

4. Har-Shai Y, Hirshowitz B. Extended upper blepharoplasty for lateral hooding of the upper eyelid using a scalpel shaped excision: a 13-year experience. Plast Reconstr Surg. 2004;113(3):1028-1035.

5. Freund RM, Nolan WB III. Correlation between brow lift outcomes and aesthetic ideals for brow height and shape in females. Plast Reconstr Surg. 1996;97(7):1343-1348.

6. Sugamata A, Yoshizawa N. Infraeyebrow excision blepharoplasty for Japanese blepharochalasis: review of 35 patients over 60 years old. Scand J Plast Reconstr Surg Hand Surg. 2010;44(1):17-20.

7. Hara T, Hara T, Narita M, Hashimoto T, Hara R, Hara T. Infero-eyebrow blepharoplasty for the upper eyelids of elderly patients. Br J Ophthalmol. 2011;95(1):109-111.

8. Ichinose A, Tahara S. Extended preseptal fat resection in Asian blepharoplasty. Ann Plast Surg. 2008;60(2):121-126.

9. Kim YS, Roh TS, Yoo WM, Tark KC, Kim J. Infrabrow excision blepharoplasty: applications and outcomes in upper blepharoplasty in Asian women. Plast Reconstr Surg. 2008;122(4):1199-1205. 
10. Ichinose A, Sugimoto T, Sugimoto I, et al. Extended infrabrow excision blepharoplasty for dermatochalasis in Asians. Arch Facial Plast Surg. 2011;13(5):327-331.

11. Vachiramon A, Aghabeigi B, Crean SJ. Eyebrow reconstruction using composite graft and microsurgical transplant. Int J Oral Maxillofac Surg. 2004;33(5):504-508.

12. Silapunt S, Goldberg LH, Peterson SR, Gardner ES. Eyebrow reconstruction: options for reconstruction of cutaneous defects of the eyebrow. Dermatol Surg. 2004;30(4 Pt 1):530-535; discussion 535.

13. Ergun SS, Sahinoglu K. Eyebrow transplantation. Ann Plast Surg. 2003;51(6):584-586.

14. Vachiramon A, Aghabeigi B, Crean SJ. Reconstruction of the eyebrow using a combination of hair-bearing composite graft and microsurgical hair follicle transplant techniques. Br J Oral Maxillofac Surg. 2003;41(5):355-357.
15. Motomura H, Muraoka M, Nose K. Eyebrow reconstruction with intermediate hair from the hairline of the forehead on the pedicled temporoparietal fascial flap. Ann Plast Surg. 2003;51(3):314-318; discussion 319-320.

16. Kim KS, Hwang JH, Kim DY, Lee SY, Cho BH. Eyebrow island flap for reconstruction of a partial eyebrow defect. Ann Plast Surg. 2002;48(3):315-317.

17. Goldman GD. Eyebrow transplantation. Dermatol Surg. 2001;27(4):352-354.

18. Fritz TM, Burg G, Hafner J. Eyebrow reconstruction with free skin and hair-bearing composite graft. J Am Acad Dermatol. 1999;41(6):1008-1010.

19. Narita M. Hair transplantation, especially as a repair of the eyebrows and eyelashes in leprous patients. Keisei Geka. 1965;8(Suppl 3):1-8. Japanese.
Clinical Ophthalmology

\section{Publish your work in this journal}

Clinical Ophthalmology is an international, peer-reviewed journal covering all subspecialties within ophthalmology. Key topics include: Optometry; Visual science; Pharmacology and drug therapy in eye diseases; Basic Sciences; Primary and Secondary eye care; Patient Safety and Quality of Care Improvements. This journal is indexed on

\section{Dovepress}

PubMed Central and CAS, and is the official journal of The Society of Clinical Ophthalmology (SCO). The manuscript management system is completely online and includes a very quick and fair peer-review system, which is all easy to use. Visit http://www.dovepress.com/ testimonials.php to read real quotes from published authors. 